\title{
Improved Prognosis and Low Failure Rate with Anticoagulation as First-Line Therapy in Mesenteric Venous Thrombosis
}

\author{
S. Salim ${ }^{1,3} \cdot$ M. Zarrouk ${ }^{1,3} \cdot$ J. Elf ${ }^{1,3} \cdot$ A. Gottsäter ${ }^{1,3} \cdot$ O. Ekberg ${ }^{2}$ • \\ S. Acosta ${ }^{1,3}$
}

Published online: 17 May 2018

(C) The Author(s) 2018

\begin{abstract}
Background Monotherapy with anticoagulation has been considered as first-line therapy in patients with mesenteric venous thrombosis (MVT). The aim of this study was to evaluate outcome, prognostic factors, and failure rate of anticoagulation as monotherapy, and to identify when bowel resection was needed.

Methods Retrospective study of consecutive patients with MVT diagnosed between 2000 and 2015.

Results The overall incidence rate of MVT was 1.3/100,000 person-years. Among 120 patients, seven died due to autopsy-verified MVT without bowel resection and 15 underwent immediate bowel resection without prior anticoagulation therapy. The remaining 98 patients received anticoagulation monotherapy, whereof $83(85 \%)$ were treated successfully. Fifteen patients failed on anticoagulation monotherapy, of whom seven underwent bowel resection and eight endovascular therapy. Endovascular therapy was followed by bowel resection in three patients. Two late bowel resections were performed due to intestinal stricture. The 30-day mortality rate was $19.0 \%$ in the former (2000-2007) and $3.2 \%$ in the latter $(2008-2015)$ part of the study period $(p=0.006)$. Age $\geq 75$ years (OR 12.4, 95\% CI [2.5-60.3]), management during the former as opposed to the latter time period (OR 8.4, 95\% CI [1.3-54.7]), and renal insufficiency at admission (OR 8.0, 95\% CI [1.2-51.6]) were independently associated with increased mortality in multivariable analysis.

Conclusions Short-term prognosis in patients with MVT has improved. Contemporary data show that monotherapy with anticoagulation is an effective first choice in MVT patients.
\end{abstract}

S. Acosta

Stefan.acosta@med.lu.se

1 Department of Clinical Sciences, Lund University, Malmö, Sweden

2 Department of Translational Medicine, Division of Medical Radiology, Lund University, Malmö, Sweden

3 Vascular Centre, Department of Cardio-Thoracic and Vascular Surgery, Skåne University Hospital, 20502 Malmö, Sweden

\section{Background}

Within the spectrum of patients presenting with acute abdominal pain, it is difficult to delineate those with mesenteric venous thrombosis (MVT). MVT is a rare but life-threatening condition which without treatment can develop into bowel ischemia, bowel gangrene, peritonitis, and death [1]. Main causes of MVT are coagulation disorders, abdominal inflammatory conditions, malignancies, and liver diseases [2]. Currently available investigations fail to identify a causal factor in about $20 \%$ of patients $[3,4]$. Improved diagnostic workup with computed tomography (CT) may possibly lead to increased detection rates and earlier diagnosis of MVT [3, 4]. Immediate 
anticoagulation therapy after diagnosis has been proposed as the first-line treatment option [5].

To prospective study, MVT is challenging due to the low incidence of the condition, and large cohort studies would provide a valuable insight into the optimal management. This retrospective study was designed to evaluate prognostic factors and trends in prognosis over time in a large cohort of patients with MVT. Secondary aims were to evaluate the failure rate with anticoagulation as monotherapy, to identify when failures occurred, and when bowel resection was needed.

\section{Methods}

Identification of all patients with MVT treated surgically or non-surgically in Malmö University Hospital between 2000 and 2015 based on the International Statistical Classification of Diseases and Related Health Problems (ICD), tenth edition, codes I81 (portal vein thrombosis [PVT] or MVT) and K55 (mesenteric ischemia), and AuriculA [6] (national quality register for anticoagulant treatment). Patient records and all CT images in patients with PVT or MVT as well as unclear cases of mesenteric ischemia were scrutinized. Patients with thrombosis in the superior mesenteric vein with or without anatomical involvement of portal or splenic veins were included in the present study. Patients diagnosed 2000-2006 have been reported upon previously [7]. The patient series was pragmatically divided at the study protocol stage into two periods, the former (2000-2007) and the latter (2008-2015), for analysis of changes in patient characteristics, risk factor profile, mode of diagnosis, and outcome. In emergencies, single-detector row CT was performed between 2000 and 2003, and multidetector row CT from 2004 and onwards [8]. Mortality data were obtained from the Swedish Population Registry. Median follow-up after diagnosis for patients with MVT was 62 (interquartile range [IQR] 24-128) months. End of follow-up was September 29, 2017. The study was approved by the Research Ethical Review Board in Lund (Dnr 2015/143).

\section{Treatment strategy}

After diagnosis of MVT with CT, the mainstay of treatment was conservative with immediate full anticoagulation with either intravenous heparin infusion or subcutaneous LMWH, full bowel rest, total parenteral nutrition, and analgesia. Patients admitted with peritonitis or rapid progression toward peritonitis underwent laparotomy and bowel resection. Patients not responding to anticoagulation underwent endovascular measures with or without local thrombolysis, and those not responding to this therapy was subjected to laparotomy. Clearly necrotic and demarcated bowels were resected and anastomosed. Bowels with unclear viability were usually evaluated at a second-look laparotomy, and bowel resections were followed by anastomoses or diverting stomas. Patients with identified transient risk factors were usually treated with oral anticoagulation for 6 months, whereas those with permanent risk factors or unidentified risk factors were prescribed lifelong anticoagulation. Up to 2014, the vitamin $\mathrm{K}$ antagonist (warfarin) was the only oral anticoagulation therapy, whereas direct-acting oral anticoagulants were gradually introduced as a treatment option from 2012.

\section{Definitions}

Primary MVT is defined as an idiopathic condition, whereas secondary MVT is defined by an identified etiologic factor. Patients with abdominal pain of less than 4-week duration were classified as having acute MVT. Those with symptoms for more than 4 weeks, but without bowel infarction, and those with asymptomatic MVT diagnosed incidentally on abdominal imaging as clinically nonsignificant findings, were defined as chronic MVT. The term thrombophilia was used as a common denominator for factors that may promote MVT, such as coagulation disorders, malignancy, previous or concomitant venous thromboembolism, and use of oral anticonceptives or estrogen substitution. The presence of inherited thrombophilia such as Factor $\mathrm{V}$ Leiden mutation and acquired thrombophilia as JAK2 V617F (janus-activated kinase gain of function substitute of valine to phenylalanine at position 617) mutation was registered. Previous cardiovascular disease was defined as previous myocardial infarction, angina pectoris, history of coronary artery bypass grafting, percutaneous coronary intervention, stroke, or transient ischemic attack. Renal insufficiency was defined as a serum creatinine level higher than $105 \mu \mathrm{mol} / \mathrm{l}(1.2 \mathrm{mg} / \mathrm{dl})$ in men and $90 \mu \mathrm{mol} / 1(1.0 \mathrm{mg} / \mathrm{dl})$ in women.

\section{Statistical analysis}

Data management and statistical analysis were performed using SPSS for Windows (SPSS, version 23.0, Chicago, Illinois, USA). Age and gender-specific total incidence rates were based on the number of patients diagnosed with MVT residing in Malmö, and expressed as number of cases per 100,000 person-years. Population data, overall and gender-specific, for Malmö in 2008 obtained from Statistics Sweden were used for calculation of incidence. Differences in proportions were evaluated using $\chi^{2}$ or Fisher's exact test. Age was expressed as median (range). Variables associated with 30-day mortality $(p<0.1)$ were further 
tested in a multivariable binary logistic regression model and expressed in terms of odds ratios (OR) with 95\% confidence interval (CI). $p<0.05$ was considered significant.

\section{Results}

\section{Incidence}

One hundred and twenty patients, 67 men and 53 women, were diagnosed with MVT from 2000 to 2015 . The overall incidence rate of MVT in Malmö was estimated to 1.3/ 100,000 person-years $(1.4 / 100,000$ person-years in men and 1.2/100,000 person-years in women).

\section{Patient characteristics}

Median age at admission was 58 (range 19-95) years. Median body mass index (BMI) was 27.5 (IQR 25.2-30.0; $n=50$ ) in men and 25.8 (IQR 23.7-33.4; $n=38$ ) in women. Acute MVT was found in $115(96 \%)$ patients, and primary and secondary MVT in $26(22 \%)$ and 94 (78\%) patients, respectively. Risk factors such as any direct injury to the vein due to disease or surgery were found in $35(29 \%)$ patients, local or systemic venous congestion in $19(16 \%)$, and thrombophilia in $72(60 \%)$. Twenty (17\%) patients had abdominal malignancies. History of previous venous thromboembolism was documented in $24(20 \%)$ patients. Among 89 tested, 39 (44\%) patients had positive tests for inherited or acquired coagulation disorder. The most common thrombophilia was activated protein $\mathrm{C}$ resistance (Factor V Leiden mutation), occurring in 22 (18\%) patients (19 in heterozygous and three in homozygous genotype). In nine patients with myeloproliferative disease, eight (89\%) were JAK-2 V617 mutation positive.

Patients diagnosed in the former period (2000-2007) were older $(p=0.013)$ and had higher proportions of abdominal malignancy $(p=0.009)$ and activated protein $\mathrm{C}$ resistance $(p=0.002)$ compared to those diagnosed in the latter period (2008-2015) (Table 1).

\section{Mode of establishing diagnosis}

During the latter time period, all patients were diagnosed by radiological imaging, in $97 \%$ of cases by CT with intravenous contrast enhancement. During this period, CT was more frequently used for MVT diagnosis compared to the former time period $(p<0.001)$ (Table 2). During the former time period, there were six autopsy-verified deaths

Table 1 Patient characteristics and risk factors for mesenteric venous thrombosis in the former (2000-2007) and the latter (2008-2015) parts of the study

\begin{tabular}{lccc}
\hline Factors & Former period $(n=58)$ & Latter period $(n=62)$ & Univariable analysis $(p$ value $)$ \\
\hline Median age (years; IQR) & $64(50-73)$ & $54(47-65)$ & 0.013 \\
Women (\%) & $27(47)$ & $42(42)$ & 0.61 \\
Acute pancreatitis (\%) & $10(17)$ & $7(11)$ & 0.35 \\
Recent abdominal surgery & $5(9)$ & $3(5)$ & 0.35 \\
Thrombophilia & $40(69)$ & $32(52)$ & 0.053 \\
History of previous venous thromboembolism & $12(21)$ & $12(19)$ & 0.86 \\
Abdominal malignancy & $15(26)$ & $5(8)$ & 0.009 \\
Positive test for inherited or acquired coagulation disorder & $20 / 36(56)$ & $19 / 53(36)$ & 0.066 \\
Activated protein C resistance (Factor V Leiden mutation) & $15 / 36(42)$ & $7 / 53(13)$ & 0.002 \\
\hline
\end{tabular}

Table 2 Mode of establishing diagnosis in the former (2000-2007) and the latter (2008-2015) parts of the study

\begin{tabular}{|c|c|c|c|}
\hline Factors & Former period $(n=58)$ & Latter period $(n=62)$ & Univariable analysis ( $p$ value) \\
\hline Autopsy frequency $(\%)$ & 25 & 12 & $<0.0001$ \\
\hline \multicolumn{4}{|l|}{ Primary mode of diagnosis } \\
\hline Autopsy & $6(10.3)$ & $0(0.0)$ & \\
\hline Computed tomography (with intravenous contrast) & $41(70.7)$ & $60(96.8)$ & \\
\hline Ultrasound & $0(0.0)$ & $2(3.2)$ & \\
\hline Operation & $11(19.0)$ & $0(0.0)$ & $<0.001$ \\
\hline Bowel resection rate (excluding autopsy cases) & $14 / 52(26.9)$ & $10(16.1)$ & 0.16 \\
\hline
\end{tabular}


in patients not undergoing bowel resection, of whom two died outside of hospital.

\section{Bowel resection and endovascular therapy}

Bowel resection rates did not differ between the two periods (Table 2). Among the 98 patients receiving anticoagulation treatment, $83(85 \%)$ were successfully treated with heparin as monotherapy without need for surgical intervention (Fig. 1). Throughout the study period, fifteen patients underwent explorative laparotomy and bowel resection without preoperative diagnosis, and another 15 patients underwent bowel resection (Fig. 2) or endovascular therapy due to failure of anticoagulation as monotherapy. Endovascular therapy was performed in eight patients, out of whom three underwent bowel resection. The two late bowel resections due to intestinal stricture were performed after 3 and 5 months, respectively, after index admission. The endovascular procedures performed were thrombolysis via the superior mesenteric artery $(n=4)$, transjugular intrahepatic portal shunt (TIPS) with stenting $(n=2)$, transjugular mechanical thrombectomy (AngioJet ${ }^{\circledR}$ device [MEDRAD, Warrendale, Pennsylvania, USA]) and thrombolysis $(n=1)$, transhepatic stenting $(n=1)$, transhepatic mechanical thrombectomy (AngioJet ${ }^{\circledR}$ ), and Fogarty catheter balloon thrombectomy $(n=1)$. Another two TIPS procedures failed. Local thrombolysis via the superior mesenteric artery was not considered first option, but was used in combination with other endovascular therapies in three patients. In the fourth patient, TIPS was not considered an option due to the advanced extent of portomesenteric venous thrombosis, and $56 \mathrm{mg}$ recombinant tissue plasminogen activator (rtPA) was continuously infused into the superior mesenteric artery over $60 \mathrm{~h}$ with success and without need of bowel resection. The sum of procedures performed exceeds eight, reflecting that a combination of techniques was often used. The median dose of thrombolytic agent, alteplase (Actilyse ${ }^{\circledR}$; Boehringer, Ingelheim, Germany), administered locally in the mesenteric circulation, was $30 \mathrm{mg}$ (range 14-56) in the four treated patients. One patient underwent a failed TIPS combined with thrombolysis via the superior mesenteric artery, complicated by perihepatic hematoma requiring explorative laparotomy for control of bleeding. Bowel resection due to late intestinal stricture was performed in two patients (Fig. 3). Lifelong anticoagulation therapy after successful non-operative management was given to $49 \%(17 / 35)$ of patients in the former period and $71 \%(34 / 48)$ in the latter $(p=0.040)$.

\section{Factors associated with 30-day mortality}

Overall 30-day mortality rate was $10.8,19.0 \%$ in the former time period versus $3.2 \%$ in the latter time period $(p=0.006)$. The 30-day mortality after surgery (bowel resection and/or endovascular therapy) was $12.5 \%(2 / 16)$ in the former period versus $7.1 \%(1 / 14)$ in the latter $(p=1.0)$. Age $\geq 75$ years, management during the former as opposed to the later time period, pancreatic malignancy, and renal insufficiency at admission were all associated with increased 30-day mortality in univariable analysis. Age $\geq 75$ years (OR 12.4, 95\% CI [2.5-60.3]), management during the former time period as opposed to the latter period (OR 8.4, 95\% CI [1.3-54.7]), and renal

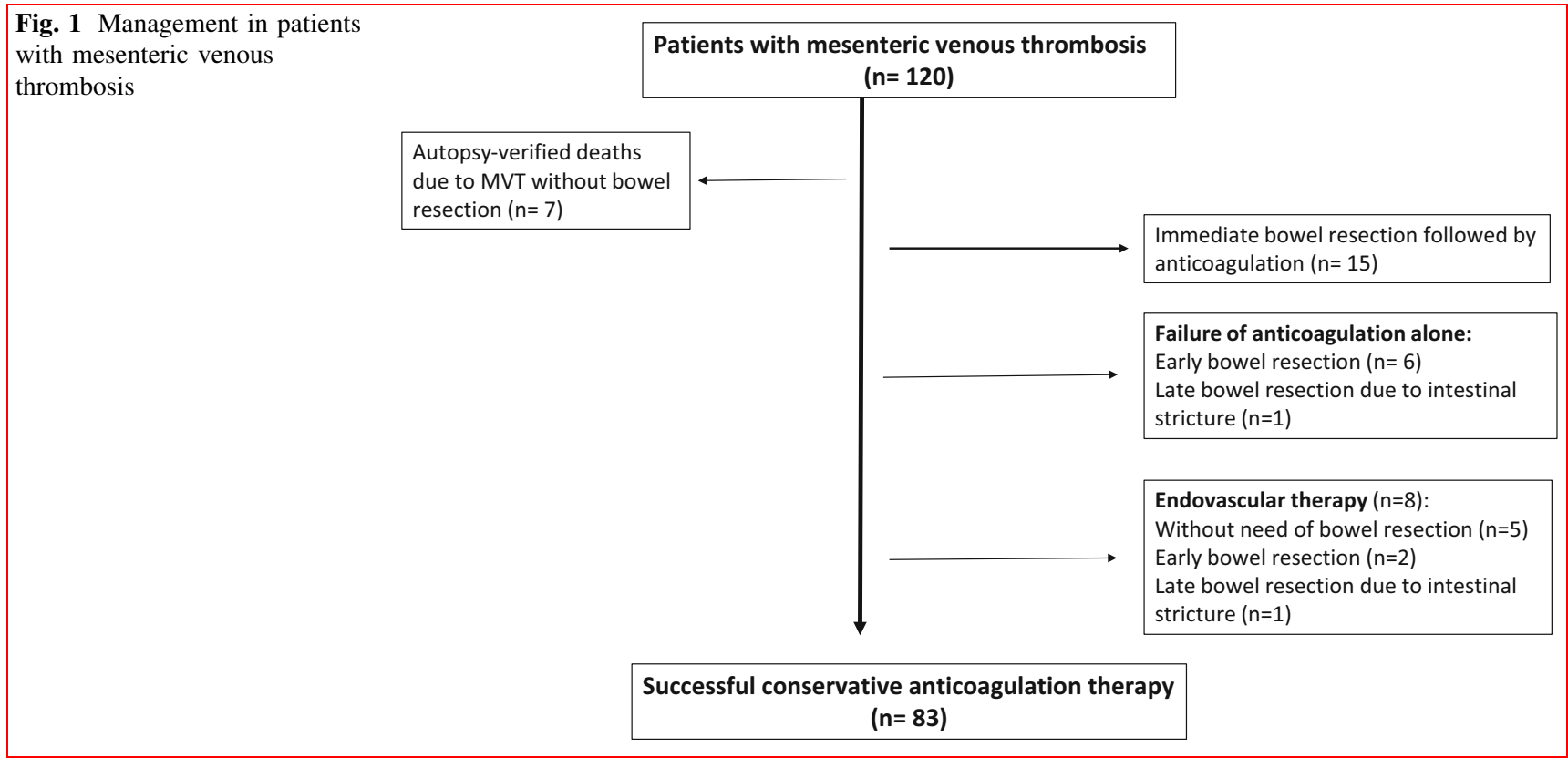



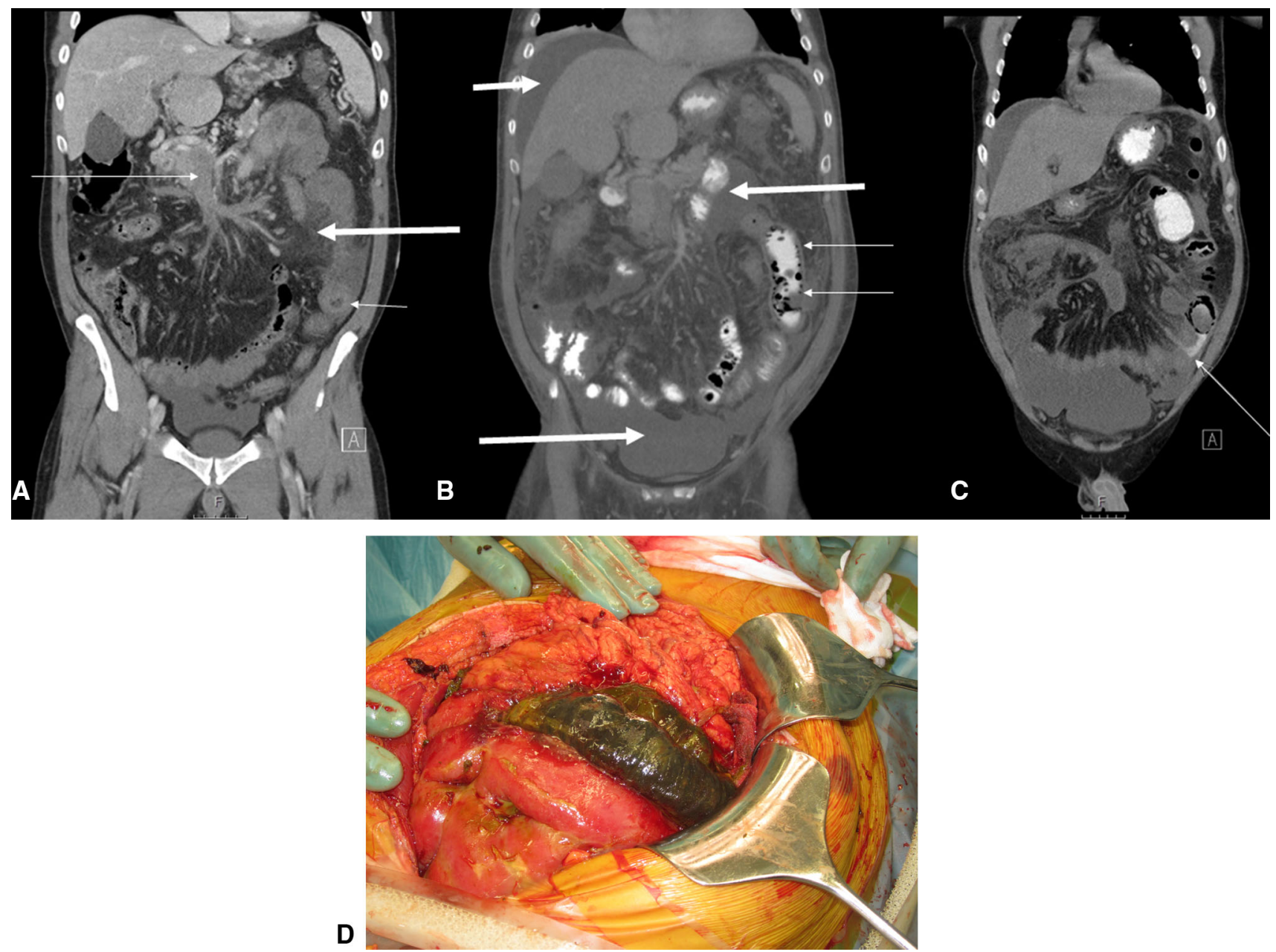

Fig. 2 Failure of anticoagulation therapy. A 50-year-old male patient with a history of ulcerative proctitis who was admitted with 3 days of abdominal pain and C-reactive protein (CRP) of $161 \mathrm{mg} / \mathrm{L}$. Diagnosis of mesenteric venous thrombosis (Fig. 2a, long thin arrow) was achieved after computed tomography (CT) with intravenous contrast enhancement and imaging in the portal/parenchymal phase. Note thickening of the jejunum (short arrow) and the mesenteric edema (long thick arrow). The patient had localized signs of peritonitis to the left in the abdomen and absent bowel sounds at the time of diagnosis. Full-dose heparin infusion was started, whereafter the patient improved temporarily but later deteriorated. A new CT (Fig. 2b) after 13 days of heparin therapy showed progression of ascites (thick arrows) and occurrence of gas bubbles (thin arrows) in the jejunal

insufficiency at admission (OR 8.0, 95\% CI [1.2-51.6]) were independently associated with increased mortality in the multivariable analysis (Table 3).

\section{Discussion}

The adjusted results of the present population-based study on 120 patients showed that prognosis for patients with MVT improved during the study period. The increased wall. Continued conservative therapy resulted in further clinical deterioration, and after 20 days of heparin therapy a CT (Fig. 2c) showed leakage of perorally administered contrast outside of the bowels (arrow). Explorative laparotomy showed a well-demarcated 1-meter-long transmural green necrosis of the jejunum (Fig. 2d) with a large perforation. The patient recovered after bowel resection, open abdomen therapy, and reanastomosis of the stapled bowel ends. Testing for thrombophilia showed that the patient was positive for JAK2 V617F mutation and a bone marrow biopsy diagnosed a polycythemia vera. The patient is scheduled for lifelong vitamin $\mathrm{K}$ antagonist therapy, and cytoreductive therapy with interferon, and is also undergoing regular venesection

diagnostic and therapeutic activity, including possibility to perform endovascular therapy, should be related to the current low 30-day mortality rate of 3.2\%. Interestingly, the proportions of patients with activated protein $\mathrm{C}$ resistance [9], abdominal malignancy [10], and age were lower in the latter period, perhaps reflecting an increased activity in preventing venous thromboembolism including prophylactic anticoagulation therapy in high-risk patients. Since randomized trials comparing safety and efficacy of various treatments most likely will be impossible to 

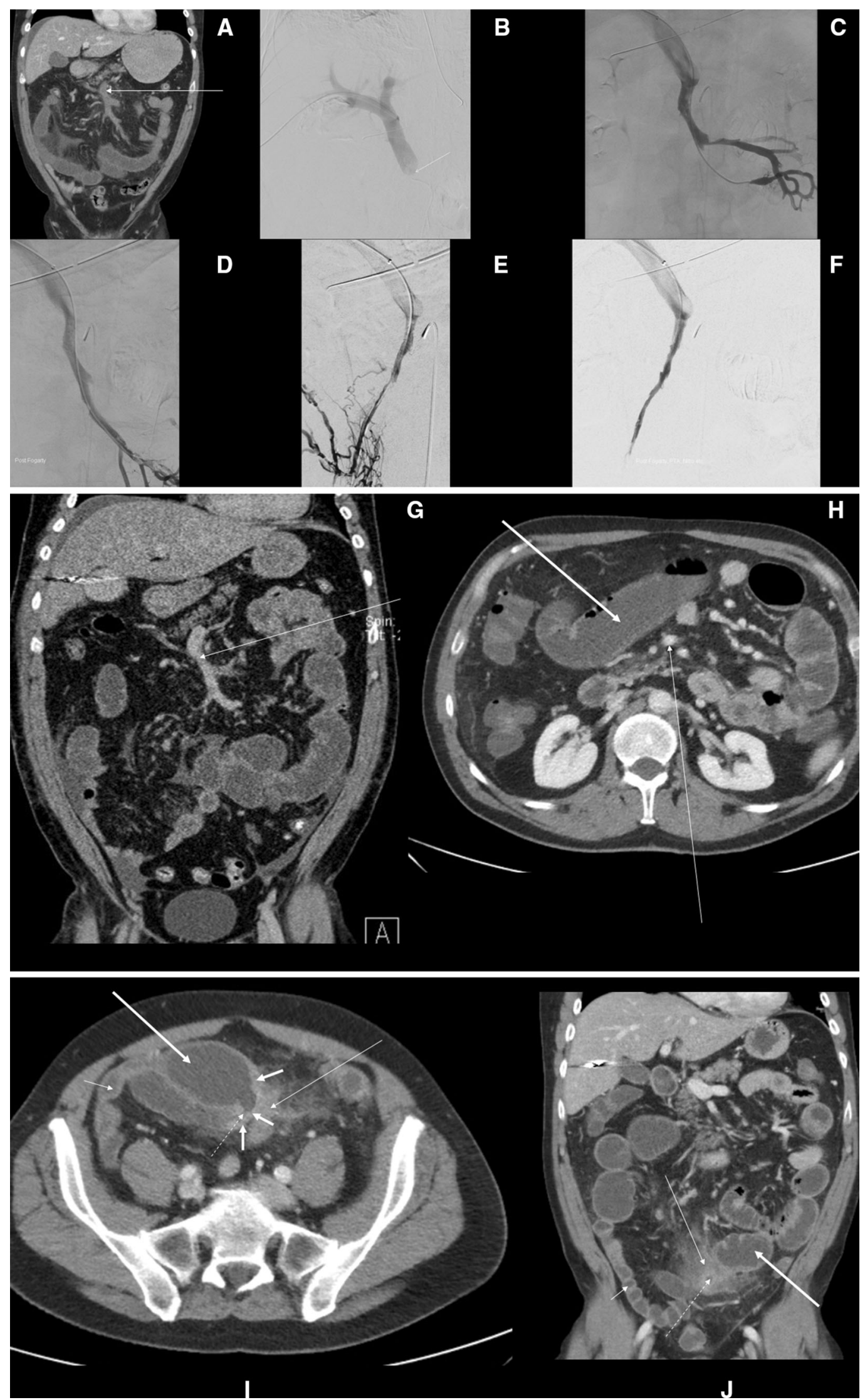
4Fig. 3 Endovascular therapy of mesenteric venous thrombosis after failure of anticoagulation treatment. A 53-year-old man with history of 3 months of anticoagulation treatment for deep venous thrombosis in the lower leg and Factor V Leiden mutation in the homozygous form. The patient fell ill with acute abdominal pain, and CT diagnosed an extensive MVT (a, arrow). He underwent transhepatic puncture and access to the portal vein. Venography showed total occlusion of the superior mesenteric vein (SMV) (b, arrow). Mechanical thrombectomy with an AngioJet ${ }^{\circledR}$ device (MEDRAD, Warrendale, Pennsylvania, USA) and endovascular Fogarty catheter thrombectomy were carried out, followed by thrombolysis with recombinant tissue plasminogen activator (rtPA) into the branches of the SMV and superior mesenteric artery. After a total dose of $25 \mathrm{mg}$ rtPA over 25 h, improved flow in the SMV was noted. Endovascular rethrombectomy with a Fogarty catheter was performed owing to residual clots in the SMV branches $(\mathbf{c}-\mathbf{f})$. CT venography before discharge showed no signs of thrombus within the SMV and the proximal parts of the major venous branches (g, arrow). The patient did not recover fully and was readmitted after 3 months with symptoms of bowel obstruction. CT venography showed fully patent SMV (h, arrow), but severe localized fibrosis in the small bowel wall (i, thick short arrows) and adjacent mesenteric fat (i, j, thin long arrow) causing a bowel stricture. Note the narrow bowel lumen at the stricture (i, $\mathbf{j}$, interrupted line). There is a prestenotic bowel dilatation (h, i, j, thick long arrow) and a poststenotic normalized bowel (i, j, thin short arrow). The patient underwent immediate bowel resection of the stricture, recovered and is on lifelong vitamin $\mathrm{K}$ antagonist medication

conduct in these patients, evidence will rely upon prospective cohort studies. International, multicenter collaboration is necessary, as exemplified by the prospective study promoted by the International Society on Thrombosis and Hemostasis (ISTH), in which affiliated centers worldwide were invited to participate [11]. In similar future studies, in which a larger proportion of patients will likely receive endovascular therapy [12], it would be preferable to not only report on therapy-related major bleeding complications, thrombotic events, bowel necrosis, and mortality. High-quality data on patency rates of the portomesenteric venous system, and patient-reported outcomes such as quality of life and pain scores before and after conservative and endovascular therapy, would also be helpful to supply physicians and patients with important data to support decision making.

The overall incidence rate of MVT in Malmö was estimated to 1.3 per 100,000 person-years, a figure in the lower range of incidence reported in the 1970s [1]. This might partly be related to the markedly reduced autopsy frequency [13], from $85 \%$ [14] to $12 \%$ in the latter time period of the present study. On the other hand, important improvements in diagnostics and treatment of hypercoagulable states have occurred during this period [15] probably resulting in a decrease in venous thromboembolism. However, since MVT is very rarely suspected already in the emergency setting [16], or sometimes confused with arterial mesenteric ischemia at laparotomy, and with the contemporary low autopsy frequency in the population the contemporary true incidence is hard to estimate.

The decrease in 30-day mortality from $19.0 \%$ during the former half of the study period to $3.2 \%$ during the latter has several explanations. Earlier diagnosis by the use of

Table 3 Factors associated with 30-day mortality in 120 patients with mesenteric venous thrombosis

\begin{tabular}{|c|c|c|c|c|c|}
\hline \multirow[t]{2}{*}{ Factors } & \multirow{2}{*}{$\begin{array}{l}\text { Number of } \\
\text { patients }\end{array}$} & \multirow{2}{*}{$\begin{array}{l}\text { 30-day mortality } \\
(\%)\end{array}$} & \multirow{2}{*}{$\begin{array}{l}\text { Univariable analysis } \\
\text { ( } p \text { value })\end{array}$} & \multicolumn{2}{|c|}{ Multivariable analysis } \\
\hline & & & & OR $(95 \% \mathrm{CI})$ & $p$ value \\
\hline All patients & 120 & 10.8 & - & & \\
\hline$\geq 75$ years & 17 & 47.1 & $<0.001^{\mathrm{a}}$ & $\begin{array}{l}12.4 \\
\quad(2.5-60.3)\end{array}$ & 0.002 \\
\hline Female gender & 53 & 17.0 & $0.054^{\mathrm{a}}$ & $2.4(0.5-11.7)$ & 0.29 \\
\hline Period (2000-2007 vs. 2008-2015) & 58 vs. 62 & 19.0 vs. 3.2 & $0.006^{\mathrm{a}}$ & $8.4(1.3-54.7)$ & 0.026 \\
\hline Malignancy & 23 & 17.4 & 0.26 & - & \\
\hline Abdominal malignancy & 20 & 20.0 & 0.15 & - & \\
\hline Pancreatic malignancy & 7 & 42.9 & $0.027^{\mathrm{a}}$ & $5.1(0.6-43.6)$ & 0.13 \\
\hline Metastatic malignancy & 14 & 28.6 & 0.045 & - & \\
\hline $\begin{array}{l}\text { History of previous venous } \\
\text { thromboembolism }\end{array}$ & 24 & 12.5 & 0.77 & - & \\
\hline Activated protein $\mathrm{C}$ resistance & $22 / 89$ & 0.0 & 1.0 & - & \\
\hline Pancreatitis & 17 & 0.0 & 0.21 & - & \\
\hline Liver cirrhosis & 6 & 33.3 & 0.13 & - & \\
\hline Inflammatory bowel disease & 7 & 0.0 & 1.0 & - & \\
\hline Renal insufficiency at admission & 20 & 25 & $0.035^{\mathrm{a}}$ & $8.0(1.2-51.6)$ & 0.029 \\
\hline Bowel resection & 24 & 8.3 & 1.0 & - & \\
\hline
\end{tabular}

${ }^{a}$ Entered into a multivariable logistic regression model 
available high-resolution, high-speed CT scanners around the clock in patients with unexplained abdominal pain would probably help to avoid development of bowel gangrene and peritonitis and the poor prognosis in these cases. CT with intravenous contrast and imaging in the portal phase is clearly the most accurate method of diagnosing the condition [17]. Corroborating other reports [18, 19], the present study showed that a non-operative approach with immediate anticoagulation therapy with unfractionated or low molecular weight heparin at the time of diagnosis was an effective treatment for acute MVT. Explorative laparotomy and bowel resection due to bowel gangrene and peritonitis will always be a way to rescue these patients in cases of rapid development of intestinal infarction, overlooked diagnosis, or late presentation as shown in Fig. 1. The clinician should also remember that the possibility of intestinal infarction is not ruled out until full resolution of pain occurs [2]. The study identified failure of anticoagulation therapy in a small proportion of patients, occurring mainly after days to weeks of medical therapy (Fig. 1).

Endovascular therapy was selectively performed in a few patients and proved to be successful in the majority of these, in whom bowel resection could be avoided. Two patients were operated after 3 and 5 months, respectively, due to late development of severe intestinal stricture with ileus (Fig. 3). CT features such as extensive thrombosis and ascites seem to be predictive factors of poor recanalization on anticoagulant therapy [20]. Clinicians should be aware of the severity of thrombotic and intestinal ischemic lesions on the $\mathrm{CT}$ images to be able to proceed with more aggressive approaches, either with endovascular therapy or laparotomy with bowel resection when needed. The 30-day mortality of $3.2 \%$ in the present study supports a conservative anticoagulation-first treatment approach (Fig. 4). Endovascular therapy may have a role in patients with extensive portomesenteric thrombosis at diagnosis, but this has to be proven in a large multicenter randomized trial.

The limitations of the present study include mainly its retrospective design. Information on bleeding complications due to anticoagulation therapy was not possible to accurately retrieve. The sample sizes of the patients in the two periods were probably not sufficiently large to be able to show a difference in bowel resection rates. Assuming that the six patients primarily diagnosed at autopsy in the former period would have undergone bowel resection if timely diagnosed, the bowel resection rate would have been significantly higher in the former compared to the latter period (20/58 vs. $10 / 62$, respectively, $p=0.02$ ). The low autopsy frequency during the latter time period might have led to an underestimation of the contemporary 30-day mortality in comparison with the former time period when autopsies were more frequently conducted. Nevertheless, the comparably large sample size in our study enabled us to evaluate our study results with multivariable testing.

In conclusion, short-term prognosis in patients with MVT seems to have improved. Contemporary data show that immediate anticoagulation is an effective first-line therapy in patients with MVT.

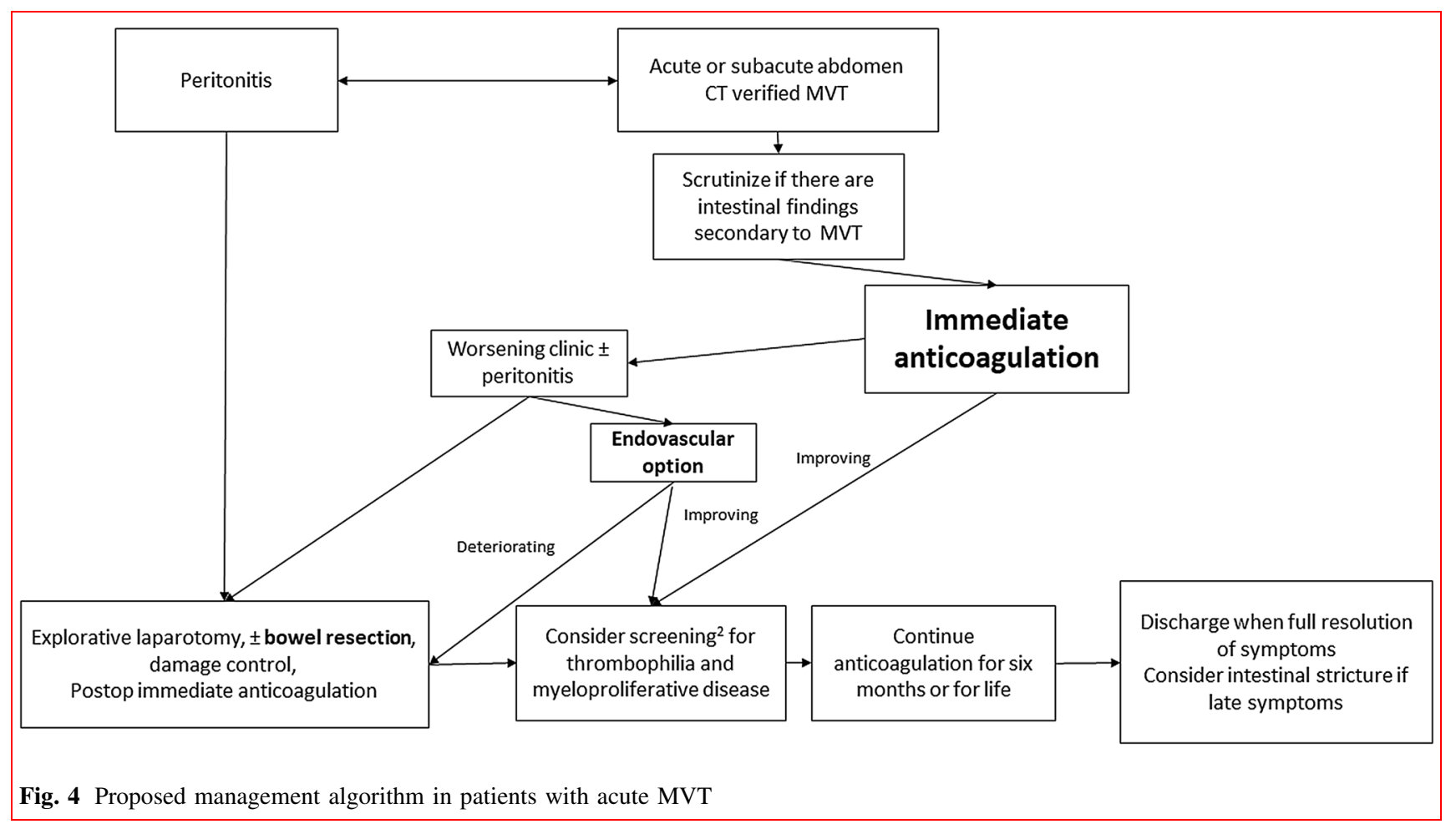


Acknowledgements Scandinavian Research Foundation for Venous Diseases.

\section{Compliance with ethical standards}

Conflict of interest The authors declared that they have no conflict of interest.

Open Access This article is distributed under the terms of the Creative Commons Attribution 4.0 International License (http://crea tivecommons.org/licenses/by/4.0/), which permits unrestricted use, distribution, and reproduction in any medium, provided you give appropriate credit to the original author(s) and the source, provide a link to the Creative Commons license, and indicate if changes were made.

\section{References}

1. Acosta S, Ögren M, Sternby N-H et al (2005) Mesenteric venous thrombosis with transmural intestinal infarction: a populationbased study. J Vasc Surg 41:59-63

2. Björck M, Koelemay M, Acosta S et al (2017) Management of the diseases of mesenteric arteries and veins. Eur J Vasc Endovasc Surg 53:460-510

3. Hamoud B, Singal AK, Kamath PS (2014) Mesenteric venous thrombosis. J Clin Exp Haematol 4:257-263

4. Acosta S, Alhadad A, Ekberg O (2009) Findings in Multi-detector row $\mathrm{CT}$ with portal phase enhancement in patients with mesenteric venous thrombosis. Emerg Radiol 16:477-482

5. Morasch MD, Ebaugh JL, Chiou AC et al (2001) Mesenteric venous thrombosis: a changing clinical entity. J Vasc Surg 34:680-684

6. Wieloch M, Själander A, Frykman V et al (2011) Anticoagulation control in Sweden: reports of time in therapeutic range, major bleeding, and thrombo-embolic complications from the national quality registry AuriculA. Eur Heart J 32:2282-2289

7. Acosta S, Alhadad A, Svensson P et al (2008) Epidemiology, risk and prognostic factors in mesenteric venous thrombosis. Br $\mathrm{J}$ Surg 10:1245-1251
8. Acosta S, Wadman M, Syk I, Elmståhl S, Ekberg O (2010) Epidemiology and prognostic factors in acute superior mesenteric artery occlusion. J Gastrointest Surg 14:628-635

9. Connors JM (2017) Thrombophilia testing and venous thrombosis. N Engl J Med 377:1177-1187

10. Hakoum MB, Kahale LA, Tsolakian IG et al (2018) Anticoagulation for the initial treatment of venous thromboembolism in people with cancer. Cochrane Database Syst Rev 1:006649

11. Ageno W, Riva N, Schulman S et al (2015) Long-term clinical outcomes of splanchnic vein thrombosis: results of an International Registry. JAMA Intern Med 175:1474-1480

12. Lerardi AM, Tsetis D, Sbaraini S et al (2017) The role of endovascular therapy in acute mesenteric ischemia. Ann Gastroenterol 30:526-533

13. Lindström P, Janzon L, Sternby N-H (1997) Declining autopsy rate in Sweden, a study of causes and consequences in Malmö, Sweden. J Intern Med 242:157-165

14. Otterhag SN, Gottsäter A, Lindblad B et al (2016) Decreasing incidence of ruptured abdominal aortic aneurysm already before start of screening. BMC Cardiovasc Disorder 16:44. https://doi. org/10.1186/s12872-016-0215-5

15. Wittens C, Davies AH, Baekgaard N et al (2015) Management of chronic venous disease: clinical practice guidelines of the european society for vascular surgery (ESVS). Eur J Vasc Endovasc Surg 49:678-737

16. Acosta S (2015) Mesenteric ischemia. Curr Opinion Crit Care 21:171-178

17. Henes F, Pickhardt P, Herzyk A et al (2017) CT angiography in the setting of suspected acute mesenteric ischemia: prevalence of ischemic and alternative diagnoses. Abdom Radiol 42:1152-1161

18. Condat B, Pessione F, Denninger M-H et al (2000) Recent portal or mesenteric venous thrombosis: increased recognition and frequent recanalization on anticoagulant therapy. Hepatology 32:466-470

19. Condat B, Pessione F, Denninger M-H et al (2001) Current outcome of portal vein thrombosis in adults: risk and benefit of anticoagulant therapy. Gastroenterology 120:490-497

20. Primignani M (2010) Portal vein thrombosis, revisited. Dig Liver Dis 42:163-170 